\title{
Disturbances in early parenting of depressed mothers and cortisol secretion in offspring: A preliminary study
}

Article

Accepted Version

Murray, L., Halligan, S. L., Herbert, J. and Goodyer, I. M. (2010) Disturbances in early parenting of depressed mothers and cortisol secretion in offspring: A preliminary study. Journal of Affective Disorders, 122 (3). pp. 218-223. ISSN 0165-0327 doi: https://doi.org/10.1016/j.jad.2009.06.034 Available at https://centaur.reading.ac.uk/17394/

It is advisable to refer to the publisher's version if you intend to cite from the work. See Guidance on citing.

To link to this article DOI: http://dx.doi.org/10.1016/j.jad.2009.06.034

Publisher: Elsevier

All outputs in CentAUR are protected by Intellectual Property Rights law, including copyright law. Copyright and IPR is retained by the creators or other copyright holders. Terms and conditions for use of this material are defined in the End User Agreement.

www.reading.ac.uk/centaur 
Central Archive at the University of Reading

Reading's research outputs online 


\section{Disturbances in Early Parenting of Depressed Mothers and Cortisol Secretion in Offspring: A Preliminary Study}

Lynne Murray (PhD), Sarah L. Halligan (DPhil), Ian Goodyer (MD,PhD) Joe Herbert (PhD),

\section{Acknowledgements}

We thank Elizabeth Gaffan, Carla Martins, Sarah Healy, Agnese Fiori-Cowley and Trish Turner for analyses of mother-child interactions.

This work was supported by grants from the Tedworth Charitable Trust and the MRC (Lynne Murray), and from the ESRC (Elizabeth Gaffan and Lynne Murray).

\section{Correspondence:}

Sarah Halligan

School of Psychology and CLS, University of Reading, Reading RG6 6AL, UK

Ph:+44(0)1183786667

Fax:+44(0)1183786665

s.l.halligan@reading.ac.uk 


\begin{abstract}
Background: Disturbances in cortisol secretion are associated with risk for psychiatric disorder, including depression. Animal research indicates that early care experiences influence hypothalamic-pituitary-adrenal (HPA) axis functioning in offspring. Similar effects are suggested in human development, but evidence of longitudinal associations between observed early parenting and offspring cortisol secretion is extremely limited. We studied associations between parenting disturbances occurring in the context of maternal postnatal depression (PND), and elevations in morning cortisol secretion in the adolescent offspring of PND mothers.
\end{abstract}

Methods: We observed maternal parenting behaviour on four occasions through the first year and at five-year follow up in postnatally depressed $(n=29)$ and well $(n=20)$ mothers. Observations were coded for maternal sensitivity and withdrawn behaviour. Basal offspring salivary cortisol secretion was measured at 13-years, using collections over 10-days.

Results: Postnatal, but not five-year, maternal withdrawal predicted elevated mean and maximum morning cortisol secretion in 13-year-old offspring. There were no significant associations between maternal sensitivity and offspring cortisol secretion.

Limitations: The sample size was relatively small, and effects tended to be reduced to trend level when covariates were considered. The correlational nature of the study (albeit longitudinal) limits conclusions regarding causality.

Conclusions: Individual differences in early maternal parenting behaviour may influence offspring cortisol secretion, and thereby risk for depression. Parenting interventions that facilitate active maternal engagement with the infant may be indicated for high risk populations.

Keywords: maternal depression, parenting, salivary cortisol, adolescents. 
A large body of research has implicated disturbances in the hypothalamic-pituitaryadrenal (HPA) axis stress response system in the development of depressive disorder. Thus, the expression of depressive disorder has been associated with elevated evening cortisol secretion and a relative lack of the negative feedback on the HPA-axis that would usually serve to keep cortisol levels in check (Gold et al., 2002). Researchers have also suggested that cortisol perturbations may represent a risk factor for the development of depression. Thus, Goodyer and colleagues studied a sample of high risk adolescents and found that the occurrence of one or more very high morning cortisol values over several days of salivary collection predicted the onset of depressive disorder in the subsequent 12-months (Goodyer et al., 2000). Similar findings have also been reported for a prospective study of a high-risk sample of women over a 1-year follow-up (Harris et al., 2000). More recently, in the currently studied sample, we found adolescents at high risk for depression (by virtue of maternal depression) to show higher morning cortisol levels than control adolescents of nondepressed mothers. Moreover, this effect mediated the association between the maternal depressive disorder and depressive symptoms in the adolescents at 16-years. Nevertheless, despite a growing evidence base to support the possible role of the HPA axis in determining risk for psychopathology, relatively little is known about the possible origins of the disturbances in cortisol secretion observed.

Developmental psychobiology research has focused on the role of early experience in determining adult functioning of the HPA axis, and associated stress responding. Rodent studies have demonstrated that early maternal tactile stimulation may influence offspring basal glucocorticoid secretion and HPA axis stress reactivity (Champagne and Meaney, 2001). Low levels of licking and grooming result in a more reactive HPA-axis and, correspondingly, increased stress responding in offspring. This effect is, at least in part, due to reduced levels of central glucocorticoid receptors (GRs) which provide negative feedback to the system (Champagne and Meaney, 2001). Non-human primate research has yielded less 
consistent findings. While a variety of severe disruptions in early care (social isolation, surrogate rearing, peer rearing) have been associated with marked behavioural disturbances in primates, alterations in HPA-axis responding have not been consistent across studies, with both elevated and depressed cortisol levels having been reported (Levine, 2005). Work focusing on less severe perturbations in the early environment has more consistently indicated HPA-axis disturbances, including disrupted diurnal rhythm following repeated maternal separations (Suomi, 1997), and elevated adrenocortical output following the imposition of foraging regimes which interfere with maternal care (Coplan et al., 2001; Lyons et al., 1998) (although for conflicting evidence see Levine and Mody, 2003).

The animal literature described above, coupled with evidence that maternal behaviour is a significant concurrent influence on human infant HPA-axis responding (Gunnar and Donzella, 2002), has stimulated a drive to establish environmental influences on human HPA-axis development. One approach has been to study the effects of severe adverse early experiences (Levine, 2005). Thus, elevated morning or daytime cortisol levels have been observed in children adopted from severely deprived institutional environments several years post adoption (Gunnar et al., 2001; Kertes et al., 2008). The severity/persistence of early deprivation appears to be a significant factor in these findings. Thus, in the Kertes et al. (2008) study, the extent of deprived care (albeit indirectly assessed by growth delay at adoption) was associated with cortisol disturbances. Elevated daytime cortisol secretion has also been observed in several samples of maltreated children and adolescents, although null findings have also been reported (Tarullo and Gunnar, 2006). While consistent with animal models, the interpretation of these findings is generally limited by a lack of evidence concerning the details of the rearing experience and, in many cases, by the presence of concurrent psychopathology (Tarullo and Gunnar, 2006).

A second approach has been to study the impact of milder parenting disturbances. In particular, researchers have examined the effects of parental depression, as a relatively 
common problem which is associated with well-documented alterations in caregiving behaviour (Field, 1984; Murray and Cooper, 2003): typically, overall sensitivity to the infant is reduced, and increased rates of withdrawn, disengaged, behaviour are found; in depressed samples characterised by socio-economic deprivation, maternal intrusive behaviour is also more common. Several studies have reported elevated basal cortisol secretion in the offspring of depressed mothers (Essex et al., 2002; Halligan et al., 2004; Hessl et al., 1998; Mannie et al., 2007), or those with bipolar disorder (Ellenbogen et al., 2006); and there is tentative evidence to suggest that maternal depression occurring early in offspring development may be particularly relevant (Essex et al., 2002; Halligan et al., 2004). However, the possible role of parenting disturbances in these effects has not been directly scrutinised. One study of offspring of parents with bipolar disorder did find reduced active, structured, parenting to predict higher basal morning cortisol levels and greater stress reactivity in offspring in late adolescence (mean age 16-years), although parenting was measured by self-report, and timing effects were not examined (Ellenbogen and Hodgins, 2009).

In a longitudinally studied sample, we found that the 13-year-old offspring of postnatally depressed mothers showed elevations in morning cortisol secretion (Halligan et al., 2004) that predicted depressive symptomatology in offspring at 16-years (Halligan et al., 2007). Furthermore, and in line with a wide literature on the effects of PND, we previously showed, in a subset of the same sample, maternal PND to be associated with early maternal parenting disturbances (reduced sensitivity to the infant, and increased withdrawal(Murray et al., 1996)). Here, in the same sample subset, we examined the nature and timing of these disturbances in maternal parenting behaviour in relation to adolescent cortisol secretion. Specifically, we investigated whether: a) there were direct associations between the quality of maternal care, particularly in the postnatal period, and cortisol secretion in adolescent offspring; and b) whether such effects could explain the associations that we have previously 
reported between maternal PND and elevated morning cortisol secretion in adolescent offspring.

Methods and Materials

\section{$\underline{\text { Sample }}$}

Participants were part of a prospective longitudinal study of the offspring of postnatally depressed and well mothers (Murray et al., 1996). A community sample of primiparous mothers was initially contacted on postnatal wards, and then finally recruited by screening for PND by postal administration of the Edinburgh Postnatal Depression Scale (Cox et al., 1987) at 6-weeks postpartum, followed by confirmatory interview with probable cases (those scoring >11): 61 women meeting Research Diagnostic Criteria (Spitzer et al., 1978) for depressive disorder were identified; 58 were recruited, along with 42 non-depressed mothers randomly selected from the same population (i.e., mothers scoring below 11 on the EPDS postal screen). A random half of the mothers in the sample $(n=49)$ completed repeated observational assessments of parenting through the first year. Participants were subsequently assessed at 5-years, 8-years, 13-years and 16-years, with retention rates above $90 \%$ at each phase. The current report examines maternal parenting assessed during the first year and at 5-years, and on salivary cortisol assessments completed at 13-years.

\section{$\underline{\text { Parenting Measures }}$}

Mothers were video-recorded in the research unit during social interactions with their infants at 2, 4, 6 and 9-months postpartum. Interactions comprised 5-minutes of face-to-face unstructured play at each age, as well as 5-minutes of play with a toy at 6 and 9 months. Maternal behaviour was scored on two dimensions; general sensitivity (characterised by appropriate, well-attuned responsiveness to the infant, including warmth, acceptance and a lack of demands on infant) and withdrawal (the mother appearing disengaged from the infant, and failing to register and respond to infant cues), using a series of standard 5-point scales (Murray et al., 1996). When children were aged 5-years, maternal behaviour was again 
assessed during a video-recorded mother-child interaction, during a refreshment period in the laboratory. Age-adjusted scales (i.e. designed to measure the same dimensions as rated in infancy, taking account of the age of the child), were used to rate maternal behaviour (Murray et al., 1999). In all cases, a higher score indicated that the behaviour was more frequently expressed. All ratings were made blind to maternal status, and inter-rater reliability was established via coding a minimum of 10 tapes (i.e., $20 \%$ of the sample) for each assessment point (intraclass correlations range 0.71-0.96). There was also a degree of stability in the parenting dimensions measured: for sensitivity, the mean intercorrelation across 2- to 9month assessments was $r=0.37$, and from infancy to 5-years it was $r=0.18$; and for withdrawal, $r=0.45$ and $r=0.17$, respectively. With regard to the independence of ratings of sensitivity and withdrawal, concurrent correlations between these two dimensions through 29 months were non-significant (range $r=0.03$ to -0.25 ), but were moderately large for the 5year assessment $(r=-0.62, P<0.001)$.

\section{Cortisol}

At 13 years, adolescents collected saliva samples (non-stimulated, passive drool) at 08:00h and 20:00h for 10 consecutive school days. Participants were instructed not to brush their teeth prior to collections, and to wash their mouths out with water 5-minutes ahead of collection time. Collection compliance was excellent, with a mean of 19/20 samples being completed. Cortisol was measured using enzyme-linked immunosorbent assay (ELISA) on $20 \mu \mathrm{l}$ samples without extraction (antibody Cambio, Cambridge UK). Samples were processed in duplicate, and intra- and inter- assay variation were $4.1 \%$ and $7.6 \%$, respectively. For each individual, mean, maximum and variability (arcsine transformed coefficient of variation) of morning and evening samples were derived, as previously reported (Halligan et al., 2004).

$\underline{\text { Additional assessments }}$ 
Possible covariates were also assessed, namely, infant birthweight, breastfeeding at 2months; and 13-year BMI and Tanner stage (Tanner, 1966), the latter being measured by adolescent self-report. In addition, adolescents completed the Mood and Feelings Questionnaire (MFQ), a validated self-report measure of depressive symptomatology (Angold et al., 1995). Covariates were selected based on previously established associations with HPA-axis functioning (Angold et al., 1999; Gold et al., 2002; Phillips et al., 1998), and also on those factors that proved to be significant in our previous studies (Halligan et al., 2004).

\section{Data Analytic Strategy}

Several of the observational maternal variables were not normally distributed, therefore non-parametric analyses (Mann-Whitney U tests or Spearman's rank correlations) were used where appropriate. First, in order to examine maternal parenting behaviour in relation to PND, Mann-Whitney tests were used to compare indices of sensitivity and withdrawal between PND and non-PND groups at each time point. Second, correlational analyses were used to examine associations between maternal parenting behaviour and adolescent cortisol indices. The fact that maternal sensitivity and withdrawal were each measured at 5 different time points resulted in the potential for a large number of correlations to be completed, and thereby the potential for Type I errors. To reduce this possibility, analyses were based on mean scores computed for postnatal parenting indices by averaging across 2- to 9-month assessments for both maternal sensitivity and maternal withdrawal, with 5-year scores being examined separately. We confirmed the validity of combining scores in this way by conducting factor analyses of sensitivity and withdrawal scores. In each case, principal components analysis yielded two factors accounting for $70 \%$ of the variance. For sensitivity, postnatal indices all loaded strongly on the first factor (loadings ranged 0.63 0.79), with only 5 -year ratings loading 0.73 on the second factor. Similarly, for maternal withdrawal, postnatal indices loaded strongly and uniquely on the first factor (range 0.72- 
0.82) with 5-year scores loading 0.90 on the second factor. Internal consistency for the combined postnatal scores was correspondingly high in both cases (Chronbach's alpha sensitivity $=0.71$, withdrawal $=0.76)$. Following preliminary correlational analyses of combined postnatal scores, we conducted post hoc analyses for individual time points only in cases where combined score effects proved significant. Finally, we used linear regression to examine: a) whether any associations between maternal behaviour and adolescent cortisol secretion were retained once possible confounds were taken account of; and b) whether maternal behaviour mediated previously reported associations between PND (Halligan et al., 2004) and adolescent morning cortisol secretion. In all cases, statistical tests were two-tailed; $P$ values of $<0.05$ were taken as being significant and $P<0.10$ as indicating a trend.

\section{Results}

Twenty control and 29 PND group dyads participated. All were Caucasian; there were no group differences in socioeconomic status, child gender, or maternal age (see Table 1).

Maternal sensitivity and withdrawal scores are presented in Figure 1. As can be seen, PND group mothers were initially less sensitive and more withdrawn, but the groups became more similar over time. Thus, index mothers were significantly less sensitive than controls at 2-month (Mann-Whitney $Z=-3.00, \mathrm{n}=49, p=.003)$ and 4-month $(Z=-2.72, \mathrm{n}=49, p=$ .007 ) assessments, but not at 6-months or 9 months, or at 5-year follow-up (all $p>.29$ ). Similarly, PND group mothers were significantly more withdrawn than controls at 2-months $(Z=2.11, \mathrm{n}=49, p=.035)$ and 4-months $(Z=2.25, \mathrm{n}=49, p=.024)$, but there were no differences at 6-months, 9-months, or 5-years (all $p>.22$ ), when maternal depression had remitted in a majority of cases (Table 1).

We conducted correlations between maternal behaviour postnatally and at 5-years, and cortisol secretion in offspring at 13 -years. For maternal sensitivity there were no significant associations with adolescent morning or evening salivary cortisol indices, for either the postnatal or 5-year observations ( $r$ s range -.10 to .09). For maternal withdrawal, the 
postnatal combined measure was positively correlated with 08:00 cortisol mean $\left(r_{s}=.40, p=\right.$ $.006)$ and maximum $\left(r_{s}=.38, p=.011\right)$ levels in adolescents, although not with day-to-day variability $\left(r_{s}=.17, \mathrm{~ns}\right)$. Post hoc analyses conducted with parenting observations from individual 2-month to 9-month time points showed positive associations between maternal withdrawn parenting and mean adolescent morning cortisol as follows: 2 -months $r_{\mathrm{s}}=0.28, P$ $=0.067$; 4-months $r_{\mathrm{s}}=0.17, P>0.10 ; 6$-months $r_{\mathrm{s}}=0.30, P=0.043 ; 9$-months $r_{\mathrm{s}}=0.32, P=$ 0.032. For maximum morning cortisol, associations with individual postnatal withdrawal measures were as follows: 2-months $r_{\mathrm{s}}=0.33, P=0.028$; 4-months $r_{\mathrm{s}}=0.19, P>0.10$; 6months $r_{\mathrm{s}}=0.27, P=0.069 ; 9$-months $r_{\mathrm{s}}=0.18, P>0.10$. In contrast, 5-year maternal withdrawal was unrelated to adolescent morning cortisol indices ( $r$ s range -.03 to -.06); and there were also no significant associations between any measure of withdrawal and evening cortisol secretion ( $r$ s range -.03 to .19$)$.

We considered the impact of potential confounds: gender, birth weight, presence / absence of breastfeeding, and adolescent BMI, pubertal status and concurrent depressive symptoms (MFQ scores). For mean 08:00 cortisol, linear regression confirmed that postnatal withdrawal was a significant predictor (beta $=0.58 \pm 0.23, \beta=0.36, p=.016$ ) when entered into the regression model alone; the addition of these six covariates to the model reduced this effect to trend level significance (beta $=0.44 \pm 0.24, \beta=0.28, p=.077$ ); however, there was no significant improvement in model fit, and none of the covariates significantly predicted 13-year mean 08:00 cortisol (all $p>0.19$ ). Similarly, for maximum 08:00 cortisol, the addition of covariates reduced a significant effect of maternal withdrawal (beta $=1.01 \pm 0.43$, $\beta=0.34, p=.024)$ to a trend (beta $=0.79 \pm 0.46, \beta=0.28, p=.096)$, but none of the covariates was a significant predictor (all $p>0.19$ ) and neither did they improve model fit.

Finally, we examined the possible role of postnatal maternal withdrawal in mediating associations between maternal PND status and adolescent 08:00 cortisol indices. For mean morning cortisol we were unable to formally test mediation according to the criteria of Baron 
and Kenny (Baron and Kenny, 1986), as linear regression indicated that our previously reported significant association between PND and elevated mean adolescent morning cortisol (Halligan et al., 2004) was at trend level in the current subset of the full sample (beta $=0.64 \pm$ $0.34, \beta=0.28, p=.062$ ). Nevertheless, we used linear regression to examine the impact of controlling for maternal PND status on the association between maternal withdrawal and mean morning cortisol; results indicated that the effect of withdrawal was retained (beta = $0.48 \pm 0.23, \beta=0.31, p=.040$ ) once maternal PND was controlled, and the latter effect was not significant (beta $=0.49 \pm 0.33, \beta=0.21$, ns; model $R^{2}=.17$ ). With regard to maximum adolescent 08:00 cortisol levels, there was an initially significant association with maternal PND (beta $=1.53 \pm 0.60, \beta=0.36, p=0.015$ ), as previously reported for the whole sample (Halligan et al., 2004), and therefore the mediating role of maternal withdrawal could be examined. In fact, the effect of PND on maximum 08.00 cortisol levels was retained when withdrawn maternal behaviour was added to the regression model (beta $=1.29 \pm 0.60, \beta=$ $0.30, p=0.038$ ), and the maternal withdrawal effect was reduced to a trend (beta $=0.76 \pm$ $0.42, \beta=0.26, p=.075 ;$ model $\left.R^{2}=.19\right)$. Thus, in total, the results were not consistent with mediation.

\section{Discussion}

In a prospective longitudinal study, we found that morning cortisol secretion in adolescent offspring was predicted by maternal parenting behaviour in the first year postpartum, but not by five-year maternal behaviour. In particular, maternal withdrawal from the infant identified during observational assessments in the first 9-months of life was associated with higher morning cortisol secretion in offspring at 13-years. We did not find similar associations between maternal behaviour at 5-years and 13-year offspring cortisol secretion.

Our data extend previous research that has shown concurrent associations between maternal behaviour and infant cortisol secretion (Kaplan et al., 2008), particularly in terms of 
infant reactivity to stress (Blair et al., 2006; Blair et al., 2008; Gunnar and Donzella, 2002). In addition, in linking direct observations of parenting to later offspring HPA axis functioning, our findings add to previous work linking environmental experiences of abuse and neglect to alterations in cortisol secretion (Tarullo and Gunnar, 2006). In this subsample of our wider study population, we could not comprehensively test the role of maternal withdrawal in mediating the relationship between maternal PND and adolescent offspring cortisol elevations; nevertheless, in the one analysis possible (i.e., for maximum cortisol), a mediational role was not identified. Although our data relate to parenting in depressed and non-depressed mothers, the conclusions are not limited to this context, since associations between maternal withdrawn behaviour and offspring cortisol secretion were broadly retained when PND status was controlled. These findings are in line with those recently reported for offspring of parents with and without bipolar disorder, where effects of self-reported parenting on adolescent cortisol still held when controlling for parental diagnosis (Ellenbogen and Hodgins, 2009). They are also consistent with data emerging from a developmental study of cortisol responding in over 1000 infants and toddlers, which found that maternal engagement during infancy predicted overall levels of cortisol secretion in the course of a challenge task in toddlerhood (Blair et al., 2008). Notably, as in our own study, these latter two reports also implicate low levels of engagement, or lack of active structuring of parenting, to be specifically associated with offspring cortisol elevations.

Our study has a number of significant strengths; in particular, these are the longitudinal design, high sample retention, the use of repeated observational assessments of maternal parenting and repeated salivary cortisol collections. Nevertheless, our sample was small, and effects were reduced to trend level when multiple covariates were considered. The interpretation of the current findings also requires caution. Although our longitudinal effects are suggestive of maternal behaviour influencing offspring cortisol secretion, findings are correlational and cannot conclusively demonstrate a causal relationship. In this regard, we 
were unable to take account of possible genetic influences which could underlie both maternal behaviour while depressed, and adolescent cortisol profile (although we would note that controlling for maternal depression did not necessarily eliminate the association between maternal withdrawal and elevated morning cortisol in offspring). Similarly, antenatal maternal depression and/or anxiety has been linked to elevated offspring cortisol secretion (O'Connor et al., 2005), and could conceivably underlie both maternal behaviour in the postnatal period and offspring cortisol perturbations in the current study (although previous research has suggested independent antenatal and postnatal effects on child outcomes, e.g., O'Connor et al., 2002). Finally, infant characteristics could also be influencing both interaction quality and subsequent cortisol secretion.

The fact that we found associations between offspring cortisol secretion and maternal withdrawal during infancy but not during childhood is intriguing, and is consistent with an early programming hypothesis and an animal literature which suggests that early environmental exposures can permanently alter HPA-axis functioning (Levine, 2005). However, strong conclusions about timing of exposures must await further research. As with all research in this field, criteria for measuring maternal behaviour must necessarily be ageadjusted. While we made every effort to ensure that the same constructs were measured over development, the lack of effects of 5-year parenting may have been a function of the inevitable variation in the exact dimensions measured. Moreover, ideally subgroups exposed to withdrawn parenting either early or late in development should be studied if effects of different timings of exposure are to be disentangled.

Although the current observations are preliminary and require replication, the implications of our own work and that of others are significant; if maternal behaviour can influence cortisol secretion in offspring in the long term, this suggests one mechanism via which risk for psychopathology is conferred to offspring, as HPA-axis disturbances have been linked to risk for depression and other affective disorders (Goodyer et al., 2001). In this 
case, interventions to facilitate better maternal engagement with her infant may be indicated in high-risk populations. 
Table 1: Sample characteristics

\begin{tabular}{|c|c|c|c|}
\hline & $\begin{array}{l}\text { Control } \\
n=20\end{array}$ & $\begin{array}{l}\text { PND } \\
n=29\end{array}$ & Comparison \\
\hline Social class I, II and III non-manual & $66.7 \%$ & $60.4 \%$ & $n s$ \\
\hline Maternal age at recruitment: $M(\mathrm{SD})$ & $28.6(3.3)$ & $28.9(4.5)$ & $n s$ \\
\hline \multicolumn{4}{|l|}{ Percent mothers currently depressed } \\
\hline - 4-months & $0 \%$ & $55.2 \%$ & $p<0.001$ \\
\hline - 6-months & $5 \%$ & $44.8 \%$ & $p<0.01$ \\
\hline - 9-months & $5 \%$ & $34.5 \%$ & $p<0.05$ \\
\hline - 5-years & $0 \%$ & $6.9 \%$ & $n s$ \\
\hline Percent male infants & $50.0 \%$ & $51.7 \%$ & $n s$ \\
\hline Birth Weight: Kg (SD) & $3.4(0.5)$ & $3.5(0.4)$ & $n s$ \\
\hline Proportion breastfed & $75.0 \%$ & $44.4 \%$ & $p<0.05$ \\
\hline 13-yr Tanner Stage 3 or above & $78.9 \%$ & $96.4 \%$ & $n s$ \\
\hline 13-yr BMI: $M(\mathrm{SD})$ & $20.1(3.8)$ & $20.6(3.5)$ & $n s$ \\
\hline 13-yr depressive symptoms: MFQ (SD) & $13.6(8.2)$ & $18.5(11.8)$ & $n s$ \\
\hline
\end{tabular}


Figure 1: Observational ratings of maternal parenting behaviours from 2-months to 5-years

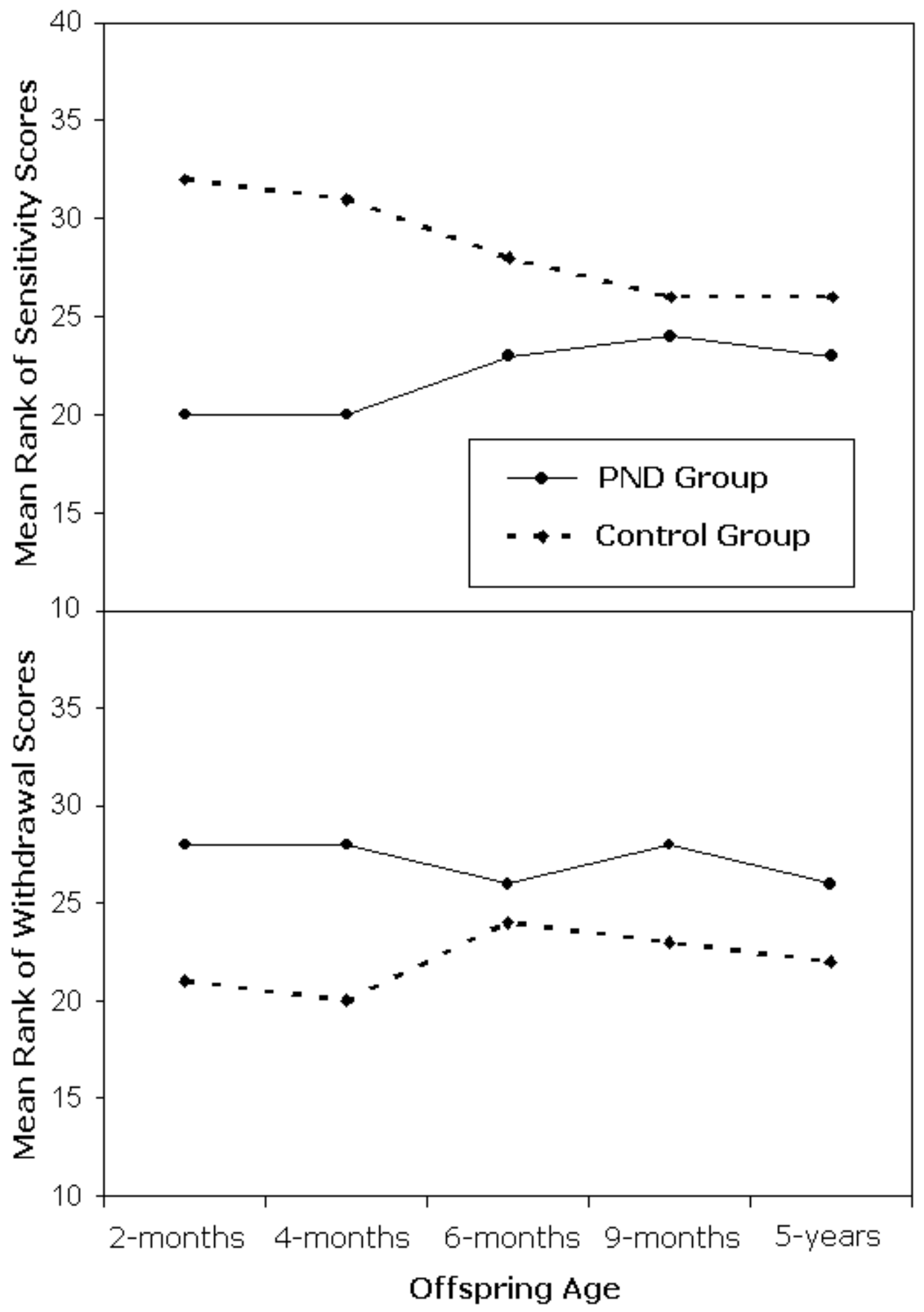




\section{Reference List}

Angold,A., Costello,E.J., Erkanli,A., Worthman,C.M., 1999. Pubertal changes in hormone levels and depression in girls. Psychol. Med. 29, 1043-1053.

Angold,A., Costello,E.J., Messer,S.C., Pickles,A., Winder,F., Silver,D., 1995. The development of a short questionnaire for use in epidemiological studies of depression in children and adolescents. Int. J. Methods Psychiatr. Res. 5, 237-249.

Baron,R.M., Kenny,D.A., 1986. The moderator-mediator variable distinction in social psychological research: conceptual, strategic, and statistical considerations. J. Pers. Soc. Psychol. 51, 1173-1182.

Blair,C., Granger,D., Willoughby,M., Kivlighan,K., 2006. Maternal sensitivity is related to hypothalamic-pituitary-adrenal axis stress reactivity and regulation in response to emotion challenge in 6-month-old infants. Ann. N. Y. Acad. Sci. 1094, 263-267.

Blair,C., Granger,D.A., Kivlighan,K.T., Mills-Koonce,R., Willoughby,M., Greenberg,M.T., Hibel,L.C., Fortunato,C.K., Family,L.P., I, 2008. Maternal and Child Contributions to Cortisol Response to Emotional Arousal in Young Children From Low-Income, Rural Communities. Dev. Psychol. 44, 1095-1109.

Champagne,F., Meaney,M.J., 2001. Like mother, like daughter: evidence for non-genomic transmission of parental behavior and stress responsivity. Prog. Brain Res. 133, 287-302.

Coplan,J.D., Smith,E.L.P., Altemus,M., Scharf,B.A., Owens,M.J., Nemeroff,C.B., Gorman,J.M., Rosenblum,L.A., 2001. Variable foraging demand rearing: sustained elevations in cisternal 
cerebrospinal fluid corticotropin-releasing factor concentrations in adult primates. Biol. Psychiatry 50, 200-204.

Cox,J.L., Holden,J.M., Sagovsky,R., 1987. Detection of postnatal depression. Development of the 10-item Edinburgh Postnatal Depression Scale. Br. J. Psychiatry 150, 782-786.

Ellenbogen,M.A., Hodgins,S., 2009. Structure provided by parents in middle childhood predicts cortisol reactivity in adolescence among the offspring of parents with bipolar disorder and controls. Psychoneuroendocrinology 34, 773-785.

Ellenbogen,M.A., Hodgins,S., Walker,C.D., Couture,S., Adam,S., 2006. Daytime cortisol and stress reactivity in the offspring of parents with bipolar disorder. Psychoneuroendocrinology $31,1164-1180$.

Essex,M.J., Klein,M.H., Cho,E., Kalin,N.H., 2002. Maternal stress beginning in infancy may sensitize children to later stress exposure: effects on cortisol and behavior. Biol. Psychiatry $52,776-784$.

Field,T., 1984. Early interactions between infants and their postpartum depressed mothers. Infant Behavior and Development 7, 537-540.

Gold,P.W., Drevets,W.C., Charney,D.S., 2002. New insights into the role of cortisol and the glucocorticoid receptor in severe depression. Biol. Psychiatry 52, 381-385.

Goodyer,I.M., Park,R.J., Netherton,C.M., Herbert,J., 2001. Possible role of cortisol and dehydroepiandrosterone in human development and psychopathology. Br. J. Psychiatry 179, 243-249. 
Goodyer,I.M., Tamplin,A., Herbert,J., Altham,P.M., 2000. Recent life events, cortisol, dehydroepiandrosterone and the onset of major depression in high-risk adolescents. Br. J. Psychiatry 177, 499-504.

Gunnar,M.R., Donzella,B., 2002. Social regulation of the cortisol levels in early human development. Psychoneuroendocrinology 27, 199-220.

Gunnar,M.R., Morison,S.J., Chisholm,K., Schuder,M., 2001. Salivary cortisol levels in children adopted from romanian orphanages. Dev. Psychopathol. 13, 611-628.

Halligan,S.L., Herbert,J., Goodyer,I., Murray,L., 2007. Disturbances in morning cortisol secretion in association with maternal postnatal depression predict subsequent depressive symptomatology in adolescents. Biol Psychiatry 62, 40-46.

Halligan,S.L., Herbert,J., Goodyer,I.M., Murray,L., 2004. Exposure to postnatal depression predicts elevated cortisol in adolescent offspring. Biol. Psychiatry 55, 376-381.

Harris,T.O., Borsanyi,S., Messari,S., Stanford,K., Brown,G.W., Cleary,S.E., Shiers,H.M., Herbert,J., 2000. Morning cortisol as a risk factor for subsequent major depressive disorder in adult women. Br. J. Psychiatry 177, 505-510.

Hessl,D., Dawson,G., Frey,K., Panagiotides,H., Self,H., Yamada,E., Osterling,J., 1998. A longitudinal study of children of depressed mothers: Psychobiological findings related to stress. In: Hann,D.M., Huffman,L.C., Lederhendler,K.K., Minecke,D.(Eds.), Advancing Research on Developmental Plasticity: Integrating the Behavioral Sciences and the Neurosciences of Mental Health. National Institutes of Mental Health, Bethseda, MD, 256. 
Kaplan,L.A., Evans,L., Monk,C., 2008. Effects of mothers' prenatal psychiatric status and postnatal caregiving on infant biobehavioral regulation: can prenatal programming be modified? Early Hum. Dev. 84, 249-256.

Kertes,D.A., Gunnar,M.R., Madsen,N.J., Long,J.D., 2008. Early deprivation and home basal cortisol levels: A study of internationally adopted children. Dev. Psychopathol. 20, 473-491.

Levine,S., Mody,T., 2003. The long-term psychobiological consequences of intermittent postnatal separation in the squirrel monkey. Neurosci. Biobehav. Rev. 27, 83-89.

Levine,S., 2005. Developmental determinants of sensitivity and resistance to stress. Psychoneuroendocrinology 30, 939-946.

Lyons,D.M., Kim,S., Schatzberg,A.F., Levine,S., 1998. Postnatal foraging demands alter adrenocortical activity and psychosocial development. Dev. Psychobiol. 32, 285-291.

Mannie,Z.N., Harmer,C.J., Cowen,P.J., 2007. Increased waking salivary cortisol levels in young people at familial risk of depression. Am J Psychiatry 164, 617-621.

Murray,L., Cooper,P.J., 2003. Intergenerational transmission of affective and cognitive processes associated with depression: infancy and the pre-school years. In: Goodyer,I.M.(Ed.), Unipolar Depression: A Lifespan Perspective. Oxford University Press, Oxford, UK, 17-46.

Murray,L., FioriCowley,A., Hooper,R., Cooper,P., 1996. The impact of postnatal depression and associated adversity on early mother-infant interactions and later infant outcome. Child Dev. 67, 2512-2526. 
Murray,L., Woolgar,M., Briers,S., Hipwell,A., 1999. Children's social representations in dolls' house play and theory of mind tasks, and their relation to family adversity and child disturbance. Social Development 8, 179-200.

O'Connor,T.G., Ben-Shlomo,Y., Heron,J., Golding,J., Adams,D., Glover,V., 2005. Prenatal anxiety predicts individual differences in cortisol in pre-adolescent children. Biol. Psychiatry 58, 211-217.

Phillips,D.I., Barker,D.J., Fall,C.H., Seckl,J.R., Whorwood,C.B., Wood,P.J., Walker,B.R., 1998. Elevated plasma cortisol concentrations: a link between low birth weight and the insulin resistance syndrome? J Clin. Endocrinol. Metab 83, 757-760.

Spitzer,R.L., Endicott,J., Robins,E., 1978. Research diagnostic criteria: rationale and reliability. Arch. Gen. Psychiatry 35, 773-782.

Suomi,S., 1997. Early determinants of behaviour: evidence from primate studies. British Medical Bulletin 53, 170-184.

Tanner,J.M., 1966. Growth at Adolescence. Appleton.

Tarullo,A.R., Gunnar,M.R., 2006. Child maltreatment and the developing HPA axis. Horm. Behav. $50,632-639$. 